\title{
Tendance et analyse des documents matériels
}

Tendency and the analysis of material objects

\section{Hélène Balfet}

\section{OpenEdition}

Journals

Édition électronique

URL : https://journals.openedition.org/tc/4992

DOI : $10.4000 /$ tc. 4992

ISSN : 1952-420X

\section{Éditeur}

Éditions de l'EHESS

\section{Édition imprimée}

Date de publication : 30 juin 2010

Pagination : $98-100$

ISSN : 0248-6016

\section{Référence électronique}

Hélène Balfet, «Tendance et analyse des documents matériels », Techniques \& Culture [En ligne], 54-55 | 2010, mis en ligne le 30 janvier 2013, consulté le 29 septembre 2022. URL : http:// journals.openedition.org/tc/4992 ; DOI : https://doi.org/10.4000/tc.4992 


\section{TENDANCE ET ANALYSE DES DOCUMENTS MATÉRIELS}

in Techniques et culture 21, 1993:27-36

Le thème abordé ici est celui auquel a été appliquée le plus classiquement la notion de tendance, et pour lequel d'ailleurs elle fut d'abord proposée par A. Leroi-Gourhan (1943 et 1945): méthode pour ordonner et analyser les faits techniques de manière à permettre des comparaisons, de manière donc à fonder leur validité comme documents ethnologiques. Elle consiste à remonter en deçà de l'acte technique, à une «tendance » qui le sous-tend mais qui n'est identifiable qu'a posteriori, lorsqu'elle est matérialisée, dans un milieu donné, par un fait personnalisé (cessant alors d'être proprement une tendance).

Pour rendre compte de l'usage que moi-même et notre groupe avons fait de cette notion dans l'analyse des documents matériels, je me propose de présenter quelques exemples illustrant non pas un système mais une démarche, qui peut revêtir des formes diverses bien qu'il s'agisse toujours de confronter logique technique et foisonnement des faits. Deux aspects de cette démarche seront illustrés chacun par deux exemples; dans tous les cas on restera au plus près des documents (précisément des objets), pour tenter d'en établir un langage descriptif homogène, soit au niveau premier de la dénomination (céramique, vêtement) soit pour rendre compte d'objets techniques complexes (métiers à tisser, vanneries).

Un des premiers problèmes auxquels j’ai été confrontée en technologie comparée, et qui a constamment occupé une grande part de mon travail, est celui des dénominations. On notera au passage l'insistance permanente mise par A. Leroi-Gourhan sur cette question, expressément dès le premier chapitre de L'Homme et la matière, puis en filigrane tout au long des deux volumes d'Évolution et techniques. Son exemple du propulseur met d'emblée en évidence ce qu'est la relation entre tendance et nom: au premier degré du fait, le «propulseur » est 
seulement défini comme « instrument destiné à augmenter la force de propulsion d'une arme de jet », avant même de prendre forme et d'être différencié de proche en proche par l'analyse.

Notre premier exemple intéresse la dénomination des formes de poterie, problème depuis longtemps familier aux chercheurs, surtout archéologues, et auquel beaucoup de contributions ont été apportées, mais en ordre dispersé. Non que le besoin d'une « terminologie standardisée » (Woodward 1936) n'ait été ressenti et même proclamé, mais parce que sans doute, chaque école devant en priorité rendre compte (nommer et décrire les documents) d'un domaine particulier, une révision générale des termes communément acceptés paraissait moins nécessaire que la recherche d'un vocabulaire approprié à la différenciation du matériel étudié.

La démarche entreprise dans le cadre du département de Technologie comparée du Musée de l'Homme prit par force une direction diamétralement opposée à celles que l'on vient évoquer. Il est clair que l'on ne pouvait prétendre partir d'un inventaire exhaustif des formes réalisées dans le monde entier et à toutes époques; le parti pris fut donc, à partir de la notion très générale de « contenant » (assimilable à une tendance commune à tous les récipients), de retenir quelques critères morphologiques pour constituer de grandes familles de formes auxquelles on puisse attribuer des noms.

Les critères reconnus opératoires, en accord avec le vocabulaire du français courant (mais en éliminant les termes à forte connotation régionale ou culturelle), furent précisément ceux qui dessinent les propriétés de l'objet dans sa fonction de contenant, c'està-dire ceux des rapports de profondeur et de diamètre, et en complément des critères de dimension; s'ajoutent, comme «formes apparentées » à chacune de ces catégories, des objets qui doivent leur nom à l'existence d'organes de préhension et/ou de versement ou à une destination particulière (Balfet et al. 1989) (fig. l).

Les formes de poterie

1. Ouvertes 2. Fermées

3. Munies de queue

4. Munies d'anse(s)

5. Munies d'appendice pour verser.

(Tableau extrait de Balfet

1992 : 195). (Fig. 1)

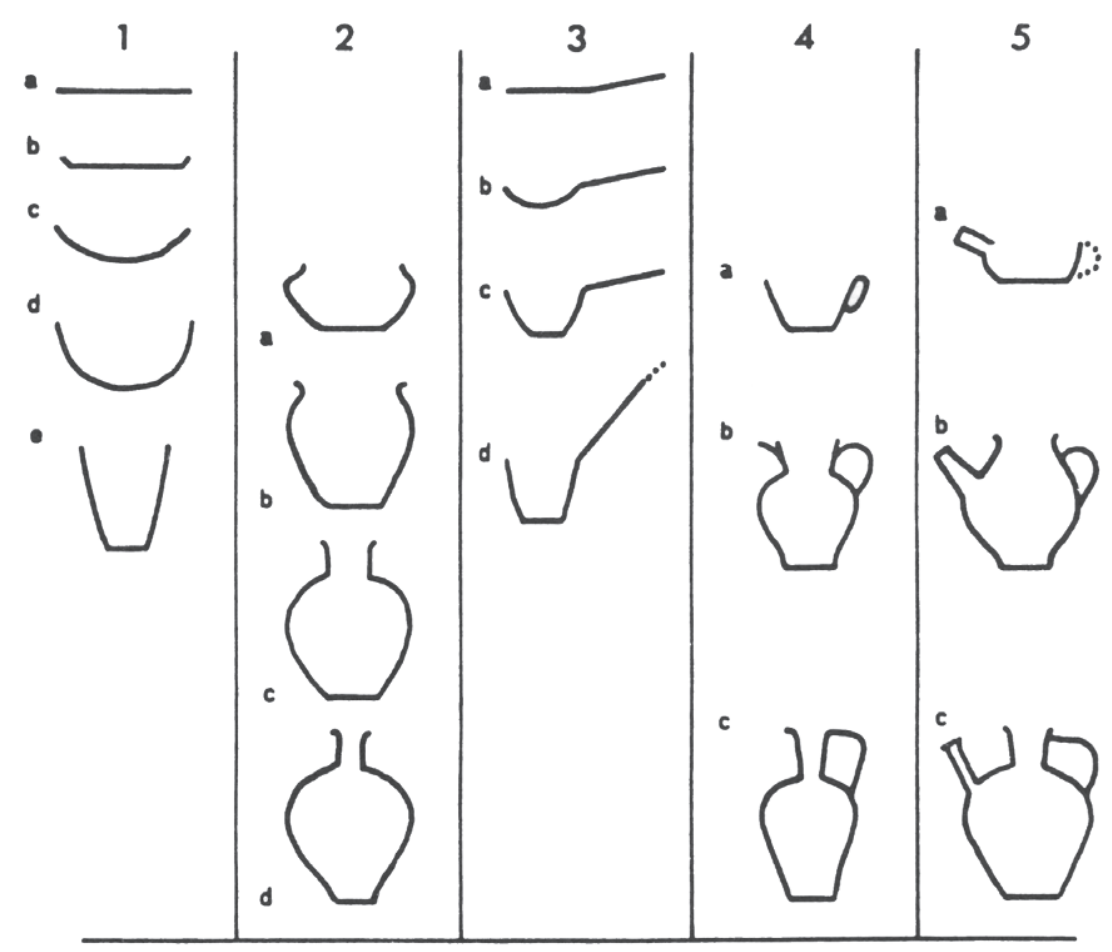


Le petit nombre des termes retenus peut surprendre. Il s'accorde, en fait, avec l'objectif expressément choisi : établir non pas un code fermé mais un système ouvert susceptible, pour l'étude d'un matériel donné, d'être complété, affiné, par la prise en compte de distinctions pertinentes dans ce contexte particulier, et d'intégrer éventuellement des noms locaux (noms vernaculaires, ou transmis par les auteurs anciens...).

Une des applications les plus démonstratives de ce système à deux niveaux fut réalisée par Guy Verron (1969) pour la classification de la céramique Sao de la région tchadienne: les 27 types reconnus sont ramenés à sept familles de formes (sous sept noms génériques), dont chacune laisse place à quelques variantes (deux à sept). Ainsi est gardée la cohérence d'une terminologie générale, sans masquer les caractères propres à l'ensemble étudié.

Une autre expérience de travail terminologique illustre l'utilité de la notion de tendance pour l'établissement de terminologie. Il s'agit d'un travail collectif visant à établir un « système descriptif » des vêtements. Comme dans l'exemple précédent, la démarche suivie se différencie nettement d'autres approches mettant pourtant en œuvre les mêmes éléments de description. Partant comme plus haut, non d'une collection déterminée mais de l'ensemble infiniment varié des pièces présentes dans un grand musée d'ethnographie, nous nous trouvons aux prises, avant même les problèmes de description, avec le problème d'une dénomination cohérente, qui ne procède ni par assimilation approximative à des formes communément désignées en français, ni par adoption de termes vernaculaires plus ou moins précisément définis.

Sur quelle base attribuer des noms à toutes les pièces de vêtement? Jusqu'à quel degré de différenciation aller pour que ces noms ordonnent la diversité des documents par larges ensembles, à partir desquels leur signalement puisse inclure des traits culturellement significatifs jusqu'à leur nom personnel d'origine? Poser la question en ces termes dicte en tout cas le sens de la démarche: elle ira de la fonction générale du vêtement, avec quelques traits dominants, jusqu'à sa forme personnalisée, marquée par le style et le contexte social d'un lieu et d'une époque (Balfet et al. 1984).

Ceci impliquait le choix de critères et surtout leur hiérarchisation, organisée en fonction de ce que nous avons considéré comme «tendance ", sous-jacente à toute élaboration vestimentaire: tendance à aménager son apparence en couvrant ou mettant en évidence telle ou telle partie du corps. Les points ou zones d'appui possibles fournissent un premier critère, ou degré du fait, de valeur universelle puisque lié aux contraintes anatomiques. Viennent ensuite quelques critères que l'on peut encore qualifier de fonctionnels, en relation avec les modalités de port, puis un ensemble de critères de forme et de structure. Enfin une série plus ou moins étendue prend en compte les matériaux, des détails de fixation et d'ornementation, assurant l'identification précise dans un contexte donné. On reconnaît le principe de l'analyse selon les degrés du fait.

Reste à décider à quel niveau peuvent être attribuées des dénominations communes à une famille de vêtements. Comme pour les formes de poterie, nous avons opté pour un petit nombre de noms génériques, termes français d'usage courant permettant de désigner des pièces vestimentaires par grands types, correspondant le plus souvent à des modalités de port. Dans notre série hiérarchique, c'est généralement, en effet, à la jonction entre les critères fonctionnels et ceux de forme et de structure que ces noms se révèlent efficaces.

Pour conclure cette première partie, on remarquera que nous rejoignons jusqu'à un certain point l'argumentation développée au sujet des classifications et de la dénomination des outils par A.-G. Haudricourt et M. J.-B. Delamarre (1955 : chap. 1): prenant position contre 
les classifications statiques et formalistes fondées sur les matériaux et sur des caractères morphologiques secondaires, ils leur opposent, pour l'ensemble des charrues et araires, le principe d'une classification qualifiée par eux de «naturelle », qui considère en premier la fonction de l'objet, à quoi il sert. Bien qu'il ne s'agisse pas pour nous de classification et bien que l'on ne puisse assimiler tendance et fonction, il n'en reste pas moins une parenté dans

\section{Concession de potière dans le village d’Alakoss, à l'est du Niger 2005}

Le parti pris fut donc, à partir de la notion très générale de «contenant» (assimilable à une tendance commune à tous les récipients), de retenir quelques critères morphologiques pour constituer de grandes familles

de formes auxquelles on puisse attribuer des noms.

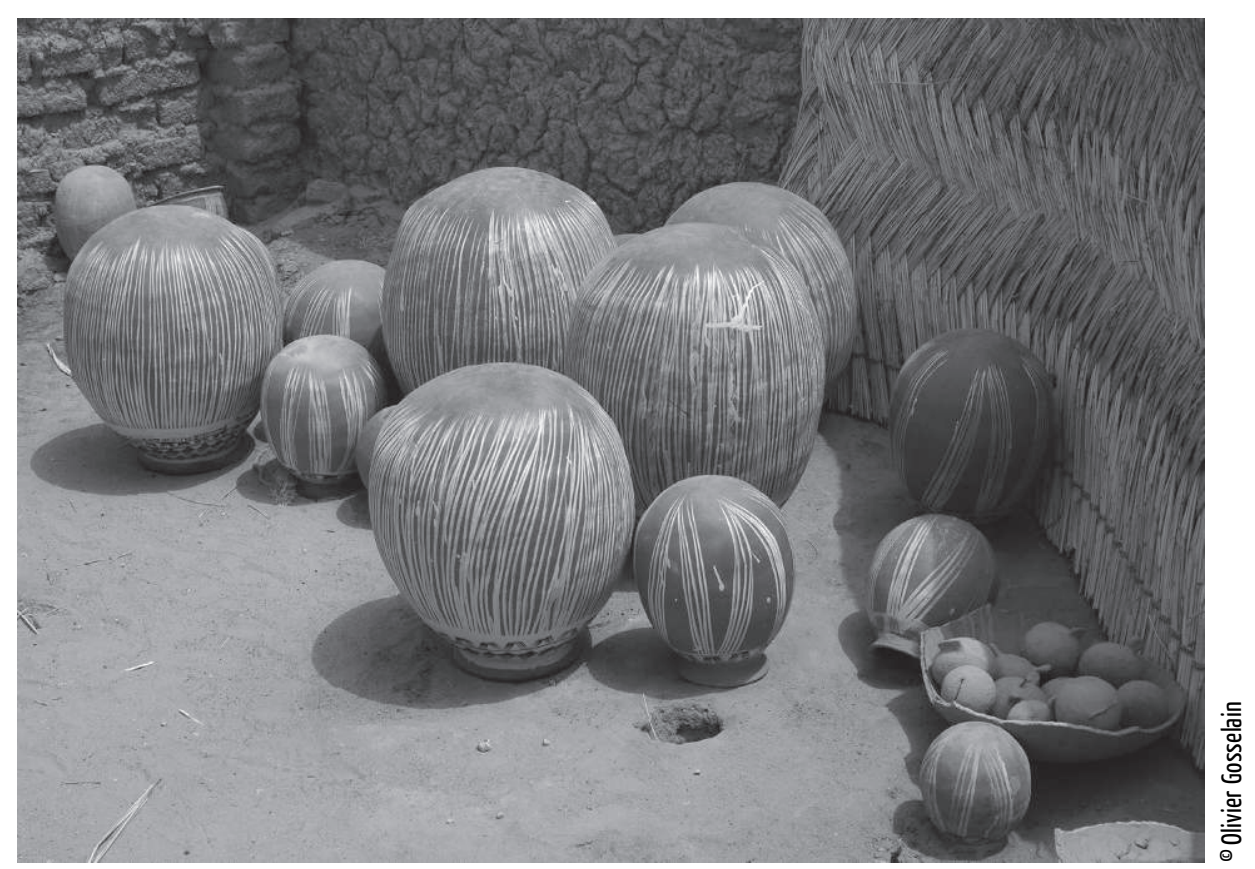

la démarche générale, qui identifie les objets par rapport à une logique technique avant de faire intervenir des ressemblances ou dissemblances morphologiques.

Un deuxième groupe de remarques, et d'exemples, concerne les problèmes particuliers posés par l'analyse d'objets techniques complexes, rebelles par là à entrer dans le schéma linéaire qui conduit par degrés depuis la tendance jusqu’au fait individualisé.

Cette difficulté m’est apparue il y a longtemps déjà, à propos des métiers à tisser, lors de confrontations entre la terminologie très fine que dictaient à Ch. Pelras, pour les métiers d'Insulinde, les distinctions qu'il était amené à établir sur la base de variantes caractéristiques de l'appartenance à telle île, telle ethnie, telle catégorie sociale, ou de la destination à telle production, et par ailleurs le regard beaucoup plus sommaire que je devais porter à ces mêmes métiers; pour moi, qui m'efforçais d'intégrer à un cadre unique tous les métiers à tisser des collections du Musée de l'Homme, ils appartenaient en fait à un même type; la question était: comment voir, décrire, ordonner un tel ensemble en donnant leur chance à toutes les variantes sans confondre les niveaux de pertinence?

Ici, rechercher une «tendance » qui sous-tende toutes ces installations ne saurait évidemment s'arrêter à la mention de l'opération «tisser », mais implique que l'on avance dans la compréhension de l'« action sur la matière » qui est en cause, de manière à reconnaître les exigences qu'elle impose aux outils correspondants. Cette recherche conduit à 
distinguer toute une série de problèmes susceptibles de recevoir, chacun, un éventail de réponses. Citons les plus évidents, auxquels toutes les installations destinées à l'activité de tissage doivent répondre (même si c'est, parfois, de façon assez sommaire), et qui sont à l'origine de leurs organes principaux:

- tendre des fils qui formeront la chaîne du tissu,

- assurer l'écartement de ces fils de chaîne pour passer la trame,

- passer le fil de trame,

- tasser régulièrement les duites (rangs de trame),

- maintenir le parallélisme des lisières...

Reconnaître ces problèmes comme autant de tendances complémentaires, et inventorier séparément, point par point, les solutions apportées à chacun permet de disposer du plus large choix de " possibles », et d'observer comment ils s'associent et se regroupent.

Ainsi voit-on se dessiner quelques grands types, surtout à partir des principales réponses aux deux premières tendances, et de leurs combinaisons. La disposition des fils entraîne la forme générale du métier, fixe ou mobile, vertical ou horizontal, à chaîne courte ou longue, étendue ou enroulée... Quant à la manière d'assurer la levée des fils, deux procédés fondamentaux dominent une grande diversité de modalités: l'un agit sur une partie des fils (un sur deux, dans le cas le plus simple d'un tissage « toile » 1/1) et sur la tension du métier pour revenir à la position initiale; l'autre agit de façon symétrique sur les deux (ou plusieurs) nappes de fils en les levant et abaissant alternativement. Dans ce dernier cas s'impose, on le conçoit, un système de levage (poulie ou balancier) au-dessus de la chaîne, et au-dessous un espace pour le jeu des pédales, c'est-à-dire un bâti qui la supporte à bonne hauteur, qui permette également de l'enrouler et de suspendre un peigne-battant pour tasser le travail. Tout ceci fournit les traits principaux d'un type de métier (" à deux rangs de lisses ») qui se reconnaît comme tel malgré un grand nombre de variantes.

Les dispositifs dissymétriques (dits « à un rang de lisses ») s'adaptent particulièrement bien aux métiers mobiles, temporairement mis en place et dont la tension se règle d'un simple coup de reins, mais ils se rencontrent aussi sur des installations fixes, à lourd bâti vertical, ou encore à chaîne longuement étendue au sol (fig. 2).

Une telle liberté d'association concerne non seulement les deux niveaux que l'on vient d'évoquer mais encore l'ensemble des solutions apportées aux problèmes rapidement inventoriés plus haut comme «tendances » complémentaires. C'est pourquoi je propose d'établir ici et dans les cas similaires un schéma pluridimensionnel qui permette de leur appliquer efficacement la méthode des degrés du fait à partir de chacune des tendances reconnues.
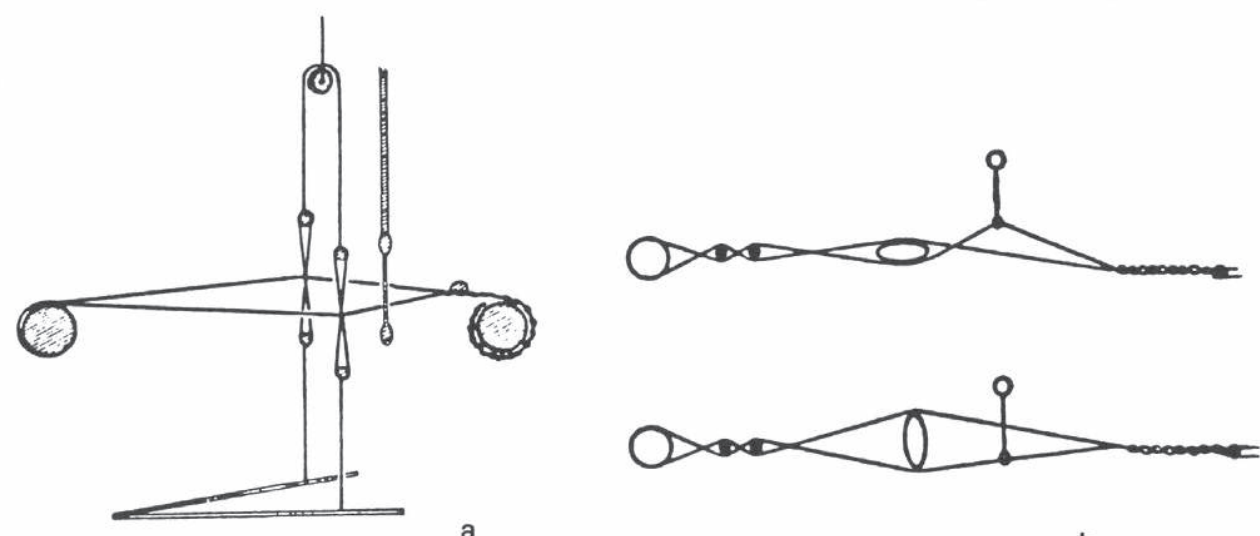

b

\section{Schémas de fonctionnement des 2 principaux types de métiers à tisser}

a) à deux rangs de lisses b) à un rang de lisses (Fig. 2) 
L'exemple des vanneries complétera celui des métiers à tisser en tant qu'objets techniques complexes. En effet ce thème, étudié de longue date dans une perspective purement technique (Balfet 1952), m’a semblé mériter d'être remis en chantier ${ }^{1}$ pour examiner l'hypothèse d'une relation entre construction, ou procédé de tressage, et mode d'expression esthétique. Il présente l'intérêt d'une homogénéité remarquable des moyens techniques disponibles, tous très limités.

Ici, la référence à une tendance générale, à construire un récipient (ou parfois une surface plane) par tressage d'éléments plus ou moins souples, paraît à première vue satisfaisante pour guider l'analyse. Néanmoins on est amené, en fait, à reconnaître une fonction différente, dans la construction de l'objet, à chacun des deux groupes d'éléments de tressage: les montants, constituant la charpente, lui donnant forme et plus ou moins de rigidité, et les brins, éléments liants, qui assemblent les premiers entre eux.

Aussi, pour ordonner le grand nombre de formules réalisées, avais-je eu recours à un tableau à deux dimensions, obtenu en croisant les deux ensembles de variables, montants et brins. Aujourd'hui, une troisième dimension doit être ajoutée pour inclure les modes d'expression esthétique, non comme élément autonome mais de telle manière que soient mises en évidence leurs relations avec les modes d'assemblage. En effet, des trois grandes options que l'on reconnaît dans la recherche esthétique appliquée aux vanneries (perfection du tressage, décors par jeux des matériaux et des armures, décors par ajout de couleurs), aucune n'est dissociable du travail de construction de l'objet.

Le tableau précédemment établi pour les deux variables techniques faisait apparaître quelques grands types mais aussi des cas rares, et des cases vides pouvant recevoir des formes non encore rencontrées. C'est à cette grille complète qu'il faut pouvoir confronter celle des effets esthétiques pour découvrir des régularités, des associations préférentielles, d'éventuelles convergences. Tout se passe, me semble-t-il, comme si une vannerie résultait du jeu croisé de deux tendances, l'une technique, amenant à faire tenir ensemble deux séries d'éléments aux fonctions complémentaires, l'autre, incluse dans le processus technique, introduisant par anticipation, d'étape en étape, les éléments grâce auxquels seront reconnues son identité et sa valeur esthétique aux yeux des fabricants et/ou des usagers.

Cette formule, où les divers caractères peuvent s'ordonner selon la logique de tendances complémentaires, me semble offrir un double avantage : à la fois permettre d'apprécier la cohérence des associations entre ces caractères, et néanmoins ne pas réduire aux quelques types les plus apparents une réalité plus riche de réalisations originales, susceptibles de poser de nouvelles questions pour une interprétation historique, ou culturelle.

Il reste, en conclusion, à faire place à la notion de critique technologique. J'entends par là la prise en compte de la tendance comme outil pour apprécier la valeur des faits matériels, en vue de leur validation comme documents ethnologiques.

La démarche d'identification de la (ou des) tendance(s) conduit, dès ce premier niveau de sa mise en œuvre, à s'interroger, face au fait observé hic et nunc, sur les conditions dans lesquelles cette solution-ci a été retenue parmi l'éventail plus ou moins ouvert des 
possibles. Voici ce fait sorti de l'anonymat (nommé) et distingué d'autres options théoriquement possibles pour les mêmes fins. Un « choix » parfois déjà significatif, ou au contraire si limité que l'objet même (ou l'action technique) devra être examiné de plus près pour livrer quelques indices utilisables. Tel rouet, tel soufflet de forge, etc., renvoient d'emblée à une zone culturelle. Par contre un tel jeu de convergences aboutit au même outil herminette, ou brasero en poterie, ou corbeille en vannerie spiralée cousue... qu'il faut se méfier de l'usage que l'on pourrait être tenté de faire de leur présence ici où là, notamment à des fins de comparaison.

C'est pourquoi, sachant l'importance de la démarche comparative dans nos disciplines, et sachant que les faits matériels sont les seuls témoins disponibles pour la plus grande partie de l'histoire humaine, il faut se donner les moyens d'assurer la validité de leur témoignage. C'est là précisément, pour une application contrôlée de la comparaison, que se révèlent les possibilités méthodologiques de ce que l'on désigne ici comme critique technologique.

La règle de base, par où elle s'inscrit dans notre propos, consiste à mesurer l'écart entre la tendance reconnue et le fait observé, c'est-à-dire d'abord à ne pas prendre l'un pour l'autre. Un exemple (caricatural) montrera en quoi peut résider la confusion. Un ourdissoir est un dispositif qui permet de constituer la chaîne d'un métier à tisser. Il en existe plusieurs modèles, mais l'étape « ourdissage » est à ce point incontournable que sa seule mention ne saurait être retenue comme un document. Pas plus que celle du pétrissage de l'argile pour la céramique.

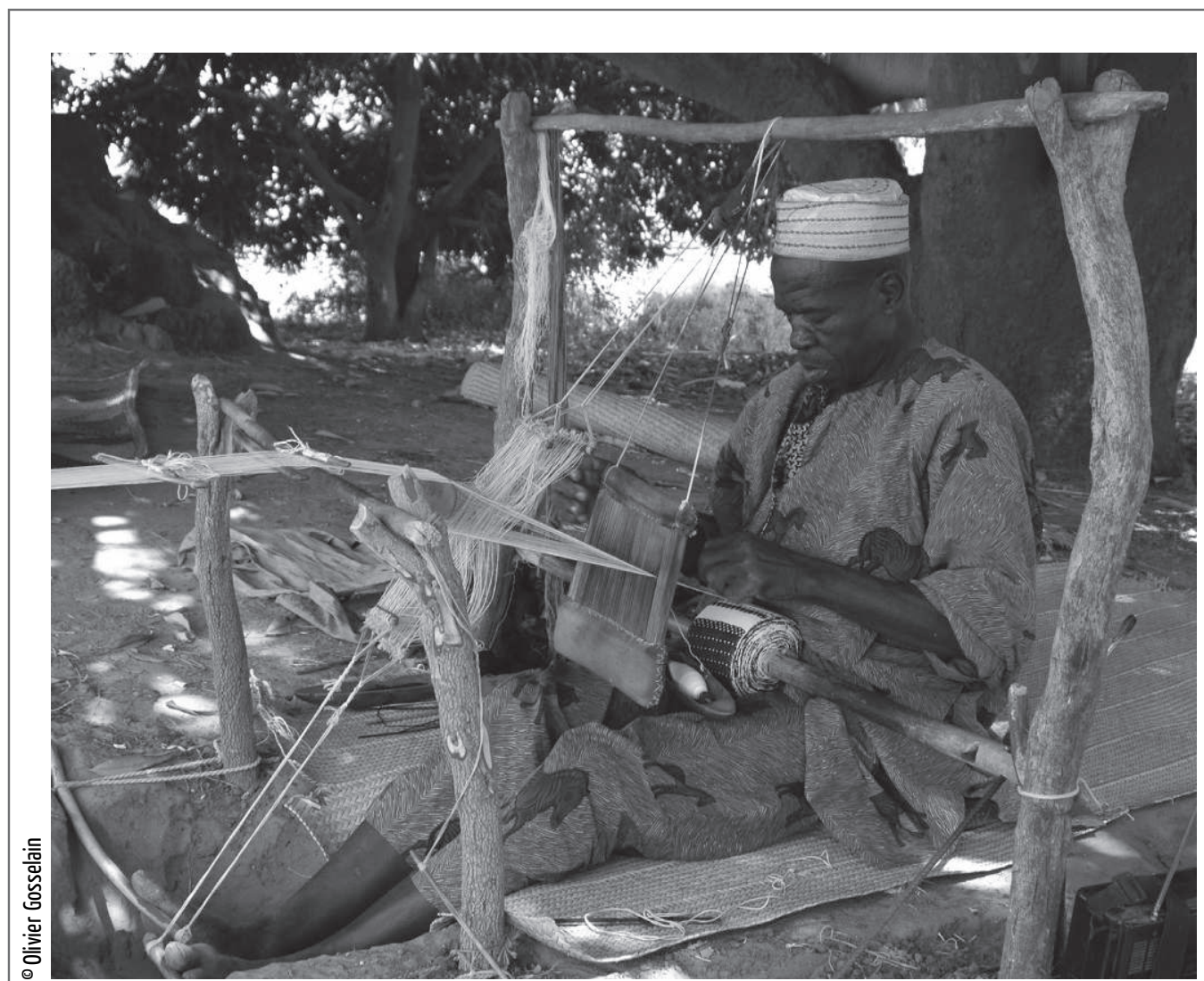

Un objet technique complexe

Comment voir, décrire, ordonner un tel objet en donnant leur chance à toutes les variantes sans confondre les niveaux de pertinence?

Métier à tisser à deux rangs de lisses, Kaw Kaw, sud-ouest du Niger.

2009 
C'est par référence à ce dernier ensemble technique que l'on tentera d'illustrer quelques modalités d'application de la démarche critique préconisée. Exemple particulièrement favorable grâce au grand nombre des variables et à l'abondance des documents mis, de longue date, à contribution comparative (bien avant ce que l'on nomme aujourd'hui ethno-archéologie).

Tout d'abord, ici comme ailleurs, il est évident que doit être tenu pour suspect tout rapprochement trait pour trait, aussi bien de produits que de procédés techniques. Les uns et les autres n'ont de sens que comme éléments d'un ensemble comprenant la totalité du milieu technique, et notamment l'inventaire complet des ustensiles, en poterie comme en d'autres matériaux (bois, métal...) fabriqués ou acquis pour répondre aux besoins de toute nature d'un groupe humain.

Mais il y a plus: cet équipement céramique lui-même tient-il, dans les sociétés mises en confrontation, une place équivalente; ou en d'autres termes résulte-t-il d'une mobilisation comparable des moyens techniques et humains disponibles, d'un même niveau d'implication de ses acteurs pour la meilleure satisfaction possible des besoins sociaux?

Cette question débouche aussitôt sur une autre concernant l'unité des productions céramiques considérées: que veut-on, que peut-on comparer, sous le même nom de poteries, parmi celles qui souvent coexistent au sein d'un même équipement? Les unes sont peut-être à la pointe des possibilités techniques de l'époque, d'autres en fort retrait, faisant figure de survivances mais tenant durablement leur place, apparemment irremplaçable... Ici, le recours à la tendance conduit à reconnaître non pas l'existence d'un mouvement vers une théorique «bonne poterie », mais une diversité de fonctions entraînant la recherche des qualités nécessaires et suffisantes pour répondre à chacune d'entre elles: des braseros et des plats pour cuisiner devront supporter de fréquents chocs thermiques, des pots destinés à tenir l'eau fraîche seront cuits au-dessous du seuil où ils cesseraient d'être poreux, pour d'autres au contraire sera recherché un maximum d'imperméabilité, avec ou sans glaçure - et dans ce cas par des procédés curieusement semblables d'un bout du monde à l'autre.

De tels exemples pourraient être multipliés, montrant des « choix » plus ou moins largement ouverts et des contraintes de tous ordres (pas seulement techniques) susceptibles d'interférer à tous les niveaux de la chaîne des degrés du fait.

Ainsi la démarche critique induite par la notion de tendance, rigoureusement appliquée à un fait matériel, peut-elle contribuer à contrôler sa fiabilité comme document ethnologique. Ainsi - mais ainsi seulement - tout fait matériel peut-il devenir document d'une ethnologie historique. 


\section{NOTES}

1. Pour contribuer à une recherche menée par le GOR 748, sur le thème: technique et esthétique.

\section{RÉFÉRENCES}

Balfet, H. 1952 La Vannerie, essai de classification, L'Anthropologie 56, 3-4: 259-280.

- 1992 La Céramique. In J. Garanger (dir.) La Préhistoire dans le monde. Paris: Presses Universitaires de France: 194-202.

Balfet, H. \& al. 1984 Un Essai de système descriptif du vêtement, L'Ethnographie LXXX: 363-373.

Balfet, H., Fauvet-Berthelot, M.-F. \& S. Monzon 1989 [1983] Lexique et typologie des poteries. Paris: Presses du CNRS

Haudricourt, A. \& M. J.-B. Delamarre 1955 L'Homme et la charrue. Paris: Gallimard.

Leroi-Gourhan, A. 1943 Évolution et Techniques I. L’Homme et la matière. Paris: Albin Michel.

— 1945 Évolution et Techniques II. Milieu et techniques. Paris: Albin Michel.

Pelras Christian, 1993 Tendance, degrés du fait et problèmes de diffusion. Exemples austronésiens, Techniques \& culture 21 : 165-194.

Verron, G. 1969 Céramique de la région tchadienne, culture Sao, Fiches typologiques africaines, Cahiers 8 et 9.

Woodward, A. 1936 Standardized terrninology, American Antiquity 2: 143-144.

\section{RÉSUMÉ}

Tendance et analyse des documents matériels. Cet article illustre d'abord l'usage classique de la notion de tendance pour l'analyse des documents matériels, pour ordonner et nommer les objets (terminologie de la céramique et du vêtement). Pour l'analyse d'objets techniques complexes (métiers à tisser, vanneries), le repérage de tendances complémentaires permet l'établissement de typologies pluridimensionnelles, tenant compte des divers regroupements de caractères. En conclusion, on évoque la notion de critique technologique, où la tendance est mise en œuvre pour évaluer la valeur ethnologique des faits matériels.

\section{ABSTRACT}

Tendency and the analysis of material objects. To start with this article gives examples of the standard use of the notion of tendency for the analysis — classifying and naming — of material objects (terminology of ceramics and clothing). For the analysis of complex objects (looms, basketwork), sorting out complementary tendancies leads to the setting up of multidimensional terminologies, taking into account diverse groupings of traits. In conclusion, the notion of technological criticism is asked, where tedency is used to evaluate the anthropological validity of material facts.

\section{MOTS CLÉS}

Objets, terminologie, typologie, critique technologique.

\section{NOTES ET RÉFÉRENCES DE l'INTRODUCTION}

1. Les lecteurs intéressés trouveront la bibliographie d'Hélène Balfet ainsi que de multiples éléments biographiques dans le volume récemment publié par F. Cousin et C. Pelras (2010) Matières, manières et sociétés. Hommage à Hélène Balfet. Aix en Provence : Publications de l'Université de Provence.
2. Lemonnier, P. 1991 De la culture matérielle à la culture? Ethnologie des techniques et préhistoire. In 25 ans d'études technologiques en préhistoire. Juanles-Pins: Éditions APDCA, 15-20. 